\title{
Efectos sobre el contexto familiar de una intervención psicosocial compleja en niños con TDAH
}

\author{
$\mathrm{M}^{\mathrm{a}}$ Jesús Presentación ${ }^{1}$, Vicente Pinto ${ }^{1}$, \\ Amanda Meliáa ${ }^{2}$ Ana Miranda ${ }^{2}$ \\ ${ }^{1}$ Universidad Jaume I, ${ }^{2}$ Universidad de Valencia
}

Disponible online 30 de agosto de 2009

\begin{abstract}
El trastorno por déficit de atención con hiperactividad ejerce una compleja influencia negativa sobre el sistema familiar pocas veces investigada. Este trabajo analiza los efectos del tratamiento psicosocial sobre aspectos amplios de la dinámica familiar, como el grado de superación de las dificultades que las familias experimentan en el plano social y económico, las mejoras en los sentimientos y actitudes de los padres, o la tensión entre los hermanos o la propia pareja. Se pretende analizar también si la presencia de problemas de conducta asociados modula los resultados de la intervención.

Participaron en el estudio un total de 42 familias distribuidas en dos grupos, con y sin tratamiento. La intervención comprendió 3 programas diferenciados en función del contexto en el que se implementan: directamente con los niños, con los padres y con los profesores. Incluyó técnicas de modificación de conducta, autorregulación, acomodaciones académicas y habilidades sociales. Los resultados ponen de manifiesto el efecto beneficioso de la intervención multicomponente implementada, sobre todo para el grupo de niños sin problemas de conducta asociados.
\end{abstract}

Palabras Clave:

TDAH, intervención psicosocial, familia

The attention deficit with hyperactivity disorder has a complex negative impact on the family system that has not been thoroughly examined. This study analyzes the effects of psychosocial treatment on a wide range of aspects of family dynamics, such as to what degree the families overcome the difficulties they experience in the social and economic areas, improvements in the feelings and attitudes of the parents, or the tension between siblings or the within the couple. The purpose is also to analyze whether the presence of associated behavioral problems modulates the effects of the intervention.

The participants in the study were 42 families divided into two groups, with and without treatment. The intervention included 3 programs differentiated according to the context in which they were implemented: directly with the children, with the parents and with the teachers. Each program included techniques of behavior modification, selfregulation, academic accommodations and social skills. The results show the beneficial effect of the multi-component intervention performed on all the family aspects analyzed, especially for the group of children without associated behavior problems.

Keywords:

ADHD, psicosocial intervention, family

Correspondencia: M. Jesús Presentación Herrero. Departamento de Psicología Evolutiva, Educativa, Social y Metodología. Facultad de Ciencias Humanas y Sociales. Universitat Jaume I. Campus del Riu Sec, 12071 Castellón (España). Telf.: 9647295 47, Fax: (34) 964729262. E-mail: presenta@psi.uji.es 
El trastorno por déficit de atención con hiperactividad (TDAH) afecta aproximadamente a un $8 \%$ de niños en el mundo, y suele ser crónico, persistiendo en la adolescencia y vida adulta en un porcentaje de casos que ronda el 50\% (Biederman y Faraone, 2005). El trastorno ha despertado un gran interés social en la última década debido, entre otras razones, a la alta prevalencia, a las implicaciones negativas que acarrea en el ámbito familiar y escolar y a la frecuente asociación que presenta con otras psicopatologías (Brown, 2003). Además, a causa de su persistencia a lo largo del curso vital y de su impacto en varias áreas de adaptación social, el TDAH constituye una pesada carga económica para la sociedad en términos de recursos necesarios para su tratamiento y de la consecuente pérdida de trabajo para los pacientes y miembros de la familia (Swensen et al., 2003).

La mayoría de expertos consideran que la heterogeneidad del TDAH sugiere vías causales complejas, de forma que los genes y el ambiente interactúan de múltiples maneras en el resultado final. La opinión general es que, si bien el trastorno es heredable en un $70-80 \%$, la forma en la que se expresa y su evolución no puede comprenderse sin considerar las experiencias que tienen lugar en los sistemas sociales, y en particular en la familia como contexto primario de socialización (Faraone et al., 2005).

Las familias con hijos con un Trastorno por déficit de atención con hiperactividad

La sintomatología definitoria del TDAH - impulsividad, hiperactividad y desatención- dificulta la labor educativa de los padres, que suelen sufrir sentimientos de frustración, culpabilidad, estrés, baja autoestima e insatisfacción con su rol parental. Los padres de niños con TDAH se consideran menos competentes en el desempeño de su papel de padres y valoran que su calidad de vida es poco satisfactoria (McLaughlin y Harrison, 2006). A menudo, están expuestos a críticas debido al comportamiento perturbador de los hijos, lo cual provoca finalmente el aislamiento social (Roselló, García, Tárraga y Mulas, 2003). Además, cuando se comparan con los padres de niños sin TDAH se sienten más deprimidos, y consideran que su función de padres les limita y condiciona en gran manera su tiempo personal (Escobar et al, 2005). Creen que sus hijos con TDAH tienen menos control afectivo y emocional, y que experimentan más dificultades para concentrarse y para adaptarse al ambiente físico y social. Asimismo muestran sentimientos fuertes de desajuste entre las expectativas que tenían y las características reales físicas y emocionales de sus hijos (Miranda, Marco y Grau, 2007).

El temperamento de los niños con TDAH junto con el estrés parental determinan significativamente el estilo de disciplina de los padres. Así, las críticas y la aplicación de procedimientos de disciplina impositivos están provocadas en gran medida por aspectos personales de los padres pero también de los niños con TDAH, que suponen una fuente de tensión en el ejercicio de la paternidad (Miranda, Rosel, Grau y Meliá, en prensa). Varios estudios empíricos han informado que las familias que tienen niños con TDAH usan métodos de disciplina poco adecuados, aplicando estrategias más agresivas y autoritarias (Miranda, Grau, Marco y Roselló, 2007). El análisis de las interacciones padres-hijos ha puesto de manifiesto que los padres que tienen hijos con TDAH emplean un tono de voz más serio y hacen comentarios más negativos sobre el comportamiento de sus hijos, imponiendo límites a veces excesivamente estrictos, pero sin ofrecer explicaciones al respecto (Lange et al., 2005).

La conclusión más relevante para la práctica que se deriva de la literatura es posiblemente la relación positiva que existe entre el estrés que produce la crianza de niños con TDAH y la aplicación en la familia de técnicas de disciplina inadecuadas, que potencian la gravedad de la sintomatología del TDAH y la aparición de trastornos de la conducta. No hay duda de que los niños con TDAH provocan altos niveles de expresión de emociones negativas en sus padres, esto es, críticas, hostilidad o sobreimplicación emocional, que se asocian con el desarrollo de problemas de conducta en la adolescencia (Taylor, Chadwick, Heptinstall, y Danckaerts, 1996). El rol de la familia en la asociación del TDAH y los problemas de conducta (PC) tiene especial interés debido al valor predictivo que los estudios de seguimiento han demostrado que dicha asociación tiene en la evolución hacia conductas adictivas y delincuencia (Mannuzza, Klein y Moulton, 2008). En este sentido los hallazgos señalan que las madres de niños con TDAH+PC hacen más comentarios negativos sobre sus hijos y despliegan menos manifestaciones físicas y verbales de afecto que las madres de los niños con sólo TDAH (Kaminski, Jones y Harshaw, 2004). La tendencia es coherente con el modelo de Patterson (1982) sobre el papel del estilo coercitivo de interacción padres/hijo en el desarrollo y mantenimiento de PC, pero en este proceso no se puede olvidar que el estrés, entendido en sentido amplio, está desempeñando un rol mediador importante.

Las consideraciones anteriores ilustran la compleja influencia negativa que el TDAH tiene en el sistema familiar. En consecuencia, las intervenciones suelen incluir un módulo de asesoramiento a padres como un componente fundamental, dentro de un paquete amplio de técnicas. Barkley (2002) ha hecho una revisión de los tratamientos psicosociales disponibles para el manejo de los niños con TDAH, llegando a la conclusión de que el entrenamiento a los padres en modificación de conducta, la utilización de estas técnicas por los profesores, las intervenciones en el plano académico y la educación especial, son las intervenciones más prometedoras para estos niños. Si a estas técnicas se les añade terapia familiar, solución de problemas y habilidades de comunicación y además todo esto se realiza en un periodo amplio de tiempo, el pronóstico de estos niños es esperanzador. Igualmente, Chronis, Jones y Raggi (2006) consideran que las intervenciones deberían integrar todos los 
componentes que han conseguido resultados positivos en el tratamiento de niños con TDAH, y todos los contextos en los que éstos se desenvuelven. En su opinión, la intervención más efectiva sería la que se lleva a cabo directamente en los contextos en los que se presentan las conductas problemáticas, incluyendo familia y escuela.

El papel mediador del entrenamiento a padres en el manejo de niños con TDAH en los resultados de la intervención ha quedado demostrado en el Multimodal Treatment Study of ADHD (MTA), la investigación más importante sobre tratamiento del trastorno realizada hasta la fecha. El único subgrupo de niños que mostró niveles normalizados de conducta perturbadora en la escuela fue aquel que: 1) recibió tratamiento combinado, esto es, medicación más modificación de conducta intensiva; y 2) sus padres mostraron una mejoría significativa de prácticas de disciplina negativas, inconsistentes e ineficaces (Hinshaw, 2007). En consonancia con estos datos, hay expertos que han argumentado que los constructos centrados en la familia pueden representar medidas más prometedoras que los síntomas para valorar los resultados de los estudios de tratamiento de niños con TDAH.

En efecto, como recoge una reciente revisión (Cunningham, 2007), un número sustantivo de investigaciones han incluido el estilo de disciplina y la autoeficacia parental entre las variables criterio para evaluar la eficacia del tratamiento. Por el contrario, pocos trabajos han examinado los efectos del tratamiento psicosocial sobre aspectos más amplios de la dinámica familiar, como el grado de superación de las dificultades que las familias experimentan en el plano social y económico, las mejoras en los sentimientos y actitudes de los padres, o en la tensión entre la pareja. Sería necesario obtener también esa misma información en relación a distintas características de los niños con TDAH. El subtipo, pero también la edad, la procedencia de la muestra o los trastornos comórbidos, son variables que pueden aportar información diferencial sobre los efectos de las intervenciones de gran importancia para las interacciones familiares.

Por consiguiente, el presente trabajo se ha planteado dos objetivos:

- En primer lugar, analizar los posibles beneficios en la familia de una intervención multicomponente desarrollada en distintos contextos con niños con TDAH, subtipo combinado (TDAH-C), sus padres y sus profesores. En concreto, se pretende profundizar en las repercusiones de la intervención a nivel de sentimientos y actitudes de los padres, vida social, economía familiar, relaciones matrimoniales, relaciones entre hermanos, así como cuestiones generales relacionadas con el grado de dificultad de vivir con su hijo con TDAH-C y la influencia que éste ejerce sobre la familia.

- En segundo lugar en esta investigación se pretende determinar si la presencia de problemas de conducta asociados al TDAH-C modula los resultados de la intervención sobre estas mismas variables familiares. Para ello se analizaran las posibles diferencias entre las percepciones de los padres de niños con TDAH-C y problemas de conducta asociados (PC) y los padres de niños con TDAH-C sin PC, antes y después del tratamiento.

\section{Método}

Para poder dar respuesta a los objetivos planteados, se partió de un diseño experimental mixto (entre-grupos e intra-grupos). En primer lugar, el total de niños con TDAH-C participantes en la investigación se dividió en dos grupos aleatoriamente, uno de ellos recibió la intervención (grupo experimental) y el otro no (grupo control). Ambos grupos fueron sometidos a dos evaluaciones, una anterior a la intervención (pre-tratamiento) y otra posterior a la misma (post-tratamiento). Para responder al segundo objetivo el grupo de niños tratados se dividió a su vez en dos subgrupos en función de la presencia/ausencia de problemas de conducta asociados.

\section{Participantes}

Participaron en esta investigación familias con niños con un diagnóstico de TDAH-C que estuvieran cursando Educación Primaria en la provincia de Castellón. Los criterios que se adoptaron para determinar la presencia de TDAH-C fueron: a) puntuación total de 6 ó más puntos en cada uno de los apartados (inatención/desorganización e hiperactividad/impulsividad) de los cuestionarios de TDAH para padres y para profesores adaptados del DSM-IV-TR (APA, 2000); b) duración de las manifestaciones superior a un año; c) aparición del trastorno antes de los 7 años; d) ausencia de retraso mental (puntuación de CI equivalente igual o superior a 80); y e) ausencia de psicosis o daño neurológico, déficit sensorial o motor. Un requisito adicional de la investigación que debían cumplir los niños con TDAH-C era no estar recibiendo en el momento de la selección ningún tratamiento farmacológico ni psicopedagógico. Para la distribución de las dos submuestras de familias con niños TDAH-C tratados, con y sin problemas de conducta, se tuvieron en cuenta los criterios diagnósticos del trastorno negativista-desafiante y del trastorno disocial del DSM-IV (APA, 1994) a través del Inventario de Síntomas para Niños y Adolescentes Stony Brook (Gadow y Sprafkin, 1995).

El total de 42 familias de niños con TDAH-C fueron distribuidas en dos grupos: experimental, con 27 niños que recibirían la intervención, y control, con 15 niños sin tratamiento. Al analizar la muestra de niños con TDAH-C tratados se encontró un predominio de varones $(85.18 \%)$ sobre mujeres (14.82\%). De forma similar, el grupo control estaba compuesto significativamente por más niños $(86.7 \%)$ que niñas $(13.3 \%)$. El rango de edad abarcaba de 7 a 10 años en ambos grupos (grupo experimental $X=8.26$; D.T. $=1.1$; grupo control $X=8.00$; D.T. $=1.20)$. En relación al CI equivalente, las medias eran de 102.4 (desviación típica 7.1) para el grupo experimental y 97.4 (desviación típica 10.0) para el control. No existían dife- 
rencias significativas entre ambos grupos en ninguna de estas variables.

Los niños con TDAH-C del grupo experimental presentaban comorbilidad con el trastorno negativista-desafiante en un $37 \%$ de los casos. Un solo caso cumplía los criterios de trastorno disocial junto con los de negativismo-desafiante, por lo que el subgrupo de TDAH-C con problemas de conducta asociados estaba compuesto por 10 niños (37\%), y el subgrupo TDAH-C sin problemas de conducta por 17 niños (63\%).

La mayor parte de los padres y de las madres de estos niños eran profesionales en activo con un estatus socioeconómico medio. La media de edad de las madres era de 42.48 años (grupo experimental) y 40.37 años (grupo control). Los padres por su parte, tenían unas medias de 44.74 años y 43.19 años respectivamente. Las familias participantes tenían entre 1 y 4 hijos, la mayoría de los padres del grupo experimental tenían 2 hijos (63.0\%), lo que ocurría en el $51.9 \%$ de los padres de los niños no tratados.

\section{Medidas}

En la selección de los participantes en la investigación se utilizaron diversos instrumentos. En primer lugar, se han aplicado dos cuestionarios que incluyen los criterios de TDAH del DSM-IV-TR (APA, 2000), para evaluar la sintomatología básica del trastorno en los contextos familiar y escolar. También se aplicaron dos subtests del WISC-R a fin de descartar casos que quedaran fuera de la normalidad intelectual. En tercer lugar, se ha utilizado también el Inventario de Síntomas para Niños y Adolescentes Stony Brook (Gadow y Sprafkin, 1995) para obtener información sobre posibles problemas de conducta asociados. Finalmente, para poder evaluar los efectos del tratamiento en el contexto familiar de los niños con TDAH se utilizó el Cuestionario de Impacto Familiar de Donenberg y Baker (1993).

Cuestionarios de TDAH adaptado del DSM-IV-TR (APA, 2000). Para la selección de la muestra, los padres y los profesores cumplimentaron el Cuestionario de TDAH adaptado del DSM-IV-TR (APA, 2000) compuestos por 18 ítems que recogen afirmaciones relativas a conductas observables que manifiestan déficit de atención ( 9 ítems) e hiperactividad /impulsividad (9 ítems).

Los padres y los profesores deben responder a la cuestión de con qué frecuencia el niño realiza estas conductas según 4 alternativas de respuesta que se corresponden con la siguiente valoración: "nunca" y "algunas veces" (0 puntos), y "bastantes veces" y "muchas veces" (1 punto). Según esta forma de valorar los ítems, el total de puntos que se puede alcanzar en cada una de las escalas (atención e hiperactividad/impulsividad) es de 9. Para establecer el diagnóstico positivo de TDAH-C y poder participar en la investigación, las valoraciones de los padres y los profesores en ambas escalas tenían que alcanzar los 6 puntos como mínimo.
Escala de Inteligencia de Wechsler para Niños Revisada, WISC-R (Wechsler, 1993). Se aplicaron 2 subpruebas del WISC-R, vocabulario y cubos, a partir de las que se puede obtener un CI equivalente (Sattler, 1982) que permite descartar un posible retraso mental, uno de los criterios de exclusión de la investigación.

Inventario de Síntomas para Niños y Adolescentes Stony Brook (Gadow y Sprafkin, 1995). Este inventario, que fue cumplimentado por los padres, está dirigido a realizar una evaluación inicial de síntomas conductuales, afectivos y cognitivos de psicopatologías de la infancia y la adolescencia. Los ítems están basados en el Manual Diagnóstico de los Trastorno Mentales DSM-IV (APA, 1994) y permiten obtener información de los padres relativa a posibles psicopatologías de sus hijos. Recoge los siguientes trastornos: negativismo-desafiante, disocial, síndrome de Tourette, ansiedad generalizada, fobia específica, fobia social, trastorno obsesivo-compulsivo, estrés postraumático, depresión mayor, distimia, ansiedad por separación, enuresis y encopresis. Para los objetivo del trabajo se han tenido en cuenta únicamente la existencia de trastorno negativista-desafiante y trastorno disocial.

Cuestionario de Impacto Familiar de Donenberg y Baker (1993). Los padres cumplimentaron en dos ocasiones el Cuestionario de Impacto Familiar de Donenberg y Baker (1993) con el objetivo de evaluar los efectos del tratamiento sobre la repercusión que los niños ejercen sobre diferentes áreas del funcionamiento familiar. Consta de 50 ítems en total, de los cuales los 48 primeros tienen 4 alternativas de respuesta (casi nunca, algunas veces, muchas veces y casi siempre). Estos 48 ítems están divididos en 5 categorías diferenciadas: 1) Sentimientos y actitudes de los padres hacia el hijo (Ej: "Mi hijo me produce sentimientos de frustración y de ira"); 2) Impacto del hijo sobre la vida social (Ej: "Me lo llevo poco de compras o a hacer recados") 3) Repercusiones de este niño en el ámbito económico (Ej: “ El costo de los servicios educativos y psicológicos es importante"); 4) Impacto del hijo sobre la relación de la pareja (Ej: "Mi hijo causa desacuerdos entre mi esposo/a y yo"); 5) Impacto del niño sobre los iguales (Ej: "Mis otros hijos no invitan a sus amigos a venir a casa debido a su mal comportamiento").

Los dos últimos ítems hacen referencia a cuestiones generales relacionadas con el grado de dificultad de vivir con su hijo y la influencia que ejerce éste sobre la familia. Ambos ítems tienen 7 alternativas de respuesta (desde mucho más fácil hasta mucho más difícil).

\section{Programa de intervención}

La intervención desarrollada, que se describe en profundidad en Miranda, Presentación, García, y Sieghentaler (2009), comprende 3 programas diferenciados en función del contexto en el que se implementan: directamente con los niños (16 sesiones), con los padres (10 sesiones) y con los profesores (10 sesiones). 
Un aspecto fundamental de la intervención es que tanto padres como profesores conocían en cada momento lo que se estaba trabajando con los niños, con el fin de facilitar la generalización de los aprendizajes. La base fundamental de este paquete de intervención, por tanto, era la comunicación entre todos los participantes. El terapeuta estaba en contacto con niños, padres y profesores en los encuentros semanales y mediante atención telefónica permanente. Así mismo, padres y profesores se mantenían en contacto diario a través de la tarjeta casa-escuela.

Programa de intervención para los niños. El programa abarcó diferentes áreas en las que los niños con TDAH-C presentan dificultades. Incluye un conjunto de técnicas cognitivoconductuales que son introducidas en pequeño grupo por un especialista: autoinstrucciones, solución de problemas, control de la ira y habilidades sociales (habilidades básicas de interacción social; habilidades sociales conversacionales; habilidades para hacer amigos; y habilidades relacionadas con sentimientos, emociones y opiniones).

La introducción de las técnicas se realiza de forma sucesiva, practicándose cada una de ellas en varias sesiones. La metodología seleccionada para implementarlas incluye procedimientos de intervención que se emplean de forma combinada en función de los objetivos perseguidos: debate, exposición, modelado (de maestría, de afrontamiento y en la corrección), juego de roles, dramatización, feedback y reforzamiento. Por último, para mantener y generalizar los aprendizajes se programan tareas y deberes fuera de las sesiones de entrenamiento.

Programa de intervención para los profesores. El curso para profesores se adaptó del programa de Miranda et al. (1999), incluyendo formación centrada en conocimientos generales sobre el TDAH (evolución diacrónica, sintomatología básica, trastornos asociados, prevalencia, pronóstico, manifestaciones evolutivas, diagnóstico, subtipos), uso de autoinstrucciones y aplicación de técnicas de modificación de conducta.

Un núcleo fundamental del curso es la técnica de autoevaluación reforzada, que pretende desarrollar en los niños las habilidades de evaluar y regular su propia conducta en el cumplimiento de las normas de clase y en la realización de sus tareas. Para potenciar la motivación de los estudiantes, la técnica incluye además un sistema de economía de puntos. El último componente del programa son las adaptaciones físicas, organizativas y metodológicas del aula a las características especiales de niños con TDAH, así como las modificaciones instruccionales en áreas concretas de conocimiento.

La instrucción de estas técnicas se realiza mediante debates, exposiciones, modelado, juego de roles, análisis de viñetas y cumplimentación de hojas de observación y de planificación de actividades.

Programa de intervención para los padres. El programa ha sido adaptado del programa para padres de Barkley (1987). Los padres aprenden a aplicar procedimientos sencillos, de manejo de contingencias, para promover la atención y la obediencia en el contexto familiar. También aprenden a reconducir sus conductas fuera del hogar y a combinar estas técnicas adaptándolas a las características de sus hijos.

Antes de comenzar con las técnicas propiamente dichas, se da a los padres información general acerca del trastorno (primera y segunda sesión), intentando modificar el pensamiento unilateral de centrar la responsabilidad del problema exclusivamente en su hijo y concienciarles de los aspectos gratificantes que conllevan las interacciones padres-hijo. Las siguientes sesiones se dedican a introducir las diversas técnicas de modificación de conducta: atención, alabanza, contacto físico, principio de Premack, recompensas, extinción, aislamiento, economía de fichas, costo de respuesta y contrato de contingencias. También durante estas sesiones los padres tienen conocimiento de las autoinstrucción y la autoevaluación reforzada.

Las últimas sesiones se centran en orientar a los padres sobre cómo apoyar el aprendizaje escolar de su hijo desde el hogar y a mejorar su salud emocional. Mediante diversas estrategias y técnicas (relajación y resolución de problemas) los padres aprenden a afrontar las situaciones complejas que les plantea la crianza de su hijo. En estas sesiones se realiza un seguimiento y profundización de las técnicas de modificación de conducta aprendidas en las sesiones anteriores y reciben también información sobre las técnicas que están aprendiendo sus hijos, concretamente, técnicas de resolución de problemas, control de la ira y habilidades sociales. En la sesión final se debate acerca de los diferentes tratamientos utilizados así como sobre la necesidad del mantenimiento del mismo y las expectativas futuras sobre sus hijos con TDAH.

La metodología seleccionada para entrenar a los padres en las diversas técnicas fue fundamentalmente el debate, la exposición y el modelado (de maestría y de afrontamiento) junto con el juego de roles, la dramatización y el análisis de viñetas que representan secuencias de aplicación de técnicas.

\section{Procedimiento}

De un total de 136 niños de 24 colegios de la provincia de Castellón que fueron seleccionados inicialmente, sólo 42 cumplían todos los requisitos establecidos en la investigación. En estos casos, se pidió a los padres la firma del consentimiento informado para que el menor fuera incluido en el estudio y la cumplimentación del Inventario de Síntomas para Niños y Adolescentes Stony Brook (Gadow y Sprafkin, 1995) y el Cuestionario de Impacto Familiar de Donenberg y Baker (1993).

Los 42 niños se dividieron después aleatoriamente en dos grupos (experimental con 27 niños y control con 15). El programa multicomponencial de intervención con los niños del 
grupo experimental se llevó a cabo fuera del horario escolar, no produciéndose ningún caso de mortandad experimental, con un porcentaje de asistencia media a todas las sesiones del $88.4 \%$. Asímismo la participación de los profesores en el curso de formación fue muy alta (93\% de las sesiones), al igual que la de los padres $(76.4 \%)$.

Una vez concluidas las intervenciones, se realizó la segunda evaluación de la misma forma y en las mismas condiciones que la inicial. Por cuestiones éticas, los padres de los niños del grupo sin tratamiento recibieron tres sesiones de 3 horas tras la evaluación del postest, en las que se les daba información general acerca del trastorno así como sobre procedimientos para controlar la conducta de sus hijos.

\section{Análisis estadísticos}

En primer lugar se realizó un estudio de normalidad de las variables. Tras analizar los datos y comprobar que no se cumplía el supuesto de normalidad y teniendo en cuenta que el tamaño muestral era reducido se consideró conveniente buscar contrastes que fueran adecuados. En este caso, los contrastes más convenientes son los no paramétricos dado que, en ellos, no se necesita hacer supuestos de distribución de los datos de la población. Se trata de pruebas menos eficientes que las paramétricas y que también llevan aparejado una mayor probabilidad de no rechazar una hipótesis nula que es falsa (incurriendo en un error de tipo II). Sin embargo, las pruebas no paramétricas han demostrado ser muy útiles como alternativa cuando no se considera apropiado el uso de pruebas paramétricas (Conover, 1999). Para analizar las comparaciones intra-grupo pre-posttratamiento se utilizó la prueba de Wilcoxon. Para las comparaciones entre los grupos se aplicó el estadístico $\mathrm{U}$ de Mann-Whitney.

\section{Resultados}

La tabla 1 recoge los resultados del pretest y postest y los análisis intragrupo pre-post de los grupos experimental y control. Como puede apreciarse en la misma las medianas del grupo tratado disminuyen tras el tratamiento, y por lo tanto la problematicidad, en todas las áreas analizadas. Los análisis comparativos entre los dos momentos muestran que esta mejoría es significativa en todos los casos (sentimientos y actitudes $Z=-4.420, p=.000$; vida social $Z=-2.735, p=.006$; economía $Z=-2.251, p=.024$; relación matrimonial $Z=-3.712$, $\mathrm{p}=.000$; relación entre hermanos $\mathrm{Z}=-3.552, \mathrm{p}=.000$; dificultad de vivir con él $Z=-2.646, p=.008$; e influencia sobre la familia $Z=-3.000, p=.003$ ). En el grupo de control, sin embargo, la situación familiar muestra intacto el impacto negativo evaluado en el pretest. No aparecen en este caso ningún tipo de modificaciones.
Tabla 1. Resultados de los grupos tratado y no tratado en el pretest y en el postest en las categorías del cuestionario de impacto familiar de Donenberg y Baker (1993).

\begin{tabular}{|c|c|c|c|c|c|c|}
\hline & \multicolumn{2}{|c|}{$\underset{\mathbf{N}=\mathbf{2 7}}{\text { TRATADO }}$} & \multicolumn{2}{|c|}{$\begin{array}{c}\text { NO TRATADO } \\
\mathbf{N}=15\end{array}$} & \multirow{2}{*}{$\begin{array}{l}\text { TRATADO } \\
- \text { pre/post }\end{array}$} & \multirow{2}{*}{$\begin{array}{c}\text { NO } \\
\text { TRATADC } \\
\text { pre/post }\end{array}$} \\
\hline & Pretest & Postest & Pretest & Postest & & \\
\hline & $\begin{array}{l}\text { Mediana } \\
\text { IQR }\end{array}$ & $\begin{array}{l}\text { Mediana } \\
\text { IQR }\end{array}$ & $\begin{array}{l}\text { Mediana } \\
\text { IQR }\end{array}$ & $\begin{array}{l}\text { Mediana } \\
\text { IQR }\end{array}$ & Z & Z \\
\hline $\begin{array}{l}\text { Sentimientos y } \\
\text { actitudes }\end{array}$ & $\begin{array}{l}8.00 \\
5.00\end{array}$ & $\begin{array}{l}4.00 \\
4.00\end{array}$ & $\begin{array}{l}6.00 \\
4.00\end{array}$ & $\begin{array}{l}6.00 \\
6.00\end{array}$ & $-4.420 * * *$ & $\mathrm{~ns}$ \\
\hline Vida social & $\begin{array}{l}1.00 \\
4.00\end{array}$ & $\begin{array}{c}.00 \\
2.00\end{array}$ & $\begin{array}{l}1.00 \\
2.00\end{array}$ & $\begin{array}{l}1.00 \\
1.00\end{array}$ & $-2.735 * *$ & $\mathrm{~ns}$ \\
\hline Economía & $\begin{array}{l}.00 \\
1.00\end{array}$ & $\begin{array}{l}.00 \\
1.00\end{array}$ & $\begin{array}{l}1.00 \\
2.00\end{array}$ & $\begin{array}{l}1.00 \\
2.00\end{array}$ & $-2.251^{*}$ & ns \\
\hline $\begin{array}{l}\text { Relación matri- } \\
\text { monial }\end{array}$ & $\begin{array}{l}2.00 \\
2.00\end{array}$ & $\begin{array}{l}.00 \\
1.00\end{array}$ & $\begin{array}{l}2.00 \\
2.00\end{array}$ & $\begin{array}{l}2.00 \\
2.00\end{array}$ & $-3.712 * * *$ & ns \\
\hline $\begin{array}{l}\text { Relación entre } \\
\text { hermanos }\end{array}$ & $\begin{array}{l}4.00 \\
3.00\end{array}$ & $\begin{array}{l}2.00 \\
3.00\end{array}$ & $\begin{array}{l}2.00 \\
2.00\end{array}$ & $\begin{array}{l}2.00 \\
1.00\end{array}$ & $-3.552 * * *$ & $\mathrm{~ns}$ \\
\hline $\begin{array}{l}\text { Dificultad de } \\
\text { vivir con él }\end{array}$ & $\begin{array}{l}1.00 \\
1.00\end{array}$ & $\begin{array}{c}.00 \\
1.00\end{array}$ & $\begin{array}{c}.50 \\
1.00\end{array}$ & $\begin{array}{l}1.00 \\
1.00\end{array}$ & $-2.646^{* *}$ & ns \\
\hline $\begin{array}{l}\text { Influencia sobre } \\
\text { la familia }\end{array}$ & $\begin{array}{l}.00 \\
1.00\end{array}$ & $\begin{array}{l}.00 \\
.00\end{array}$ & $\begin{array}{l}.00 \\
1.00\end{array}$ & $\begin{array}{l}.00 \\
1.00\end{array}$ & $-3.000 * *$ & ns \\
\hline
\end{tabular}

$*=\mathrm{p}<.05 ; * *=\mathrm{p}<.01 ; * * *=\mathrm{p}<.001$

Las comparaciones entre ambos grupos de niños muestran diferencias significativas en el pretest en relaciones entre hermanos $(\mathrm{U}=81.000, \mathrm{p}=.040)$, manifestándose en el grupo tratado significativamente más conflictivas. En el postest, por el contrario, con la mejoría en las relaciones experimentadas por el grupo tratado desaparecen estas diferencias y además ambos grupos aparecen como significativamente diferentes, en el sentido de mejora de la situación familiar en el grupo que ha recibido tratamiento, en sentimientos y actitudes de los padres $(\mathrm{U}=114.500, \mathrm{p}=.020)$, en las relaciones matrimoniales $(\mathrm{U}=66.500, \mathrm{p}=.000)$ y también en la influencia negativa que el niño con TDAH ejerce sobre la familia $(\mathrm{U}=135.000, \mathrm{p}=.002)$.

La tabla 2 muestra los efectos de la intervención para las familias de niños con TDAH-C sin y con PC asociados. Los resultados encontrados en las familias de los niños TDAH-C sin PC muestran diferencias significativas en todas las variables analizadas. Es decir, estas familias mejoran significativamente en Sentimientos y actitudes $(Z=-3.643 ; p=000)$; vida social $(Z=-2.555 ; \mathrm{p}=011)$; impacto económico $(Z=-2.070$; $\mathrm{p}=038)$; relación matrimoniales $(\mathrm{Z}=-2.910 ; \mathrm{p}=004)$; relaciones entre hermanos $(Z=-2.965 ; p=003)$; dificultad de vivir con él $(Z=-2.236 ; p=025)$; e influencia negativa sobre la familia $(\mathrm{Z}=-2.000 ; \mathrm{p}=046)$. 
Tabla 2. Resultados de los subgrupos TDAH-C tratados, con y sin PC, en el prestest y el postest en las categorías del cuestionario de impacto familiar de Donenberg y Baker (1993).

\begin{tabular}{|c|c|c|c|c|c|c|}
\hline & \multicolumn{3}{|c|}{ 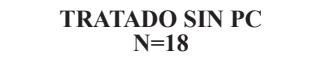 } & \multicolumn{3}{|c|}{$\begin{array}{c}\text { TRATADO CON PC } \\
\mathbf{N}=9\end{array}$} \\
\hline & Pretest & Postest & & Pretest & Postest & \\
\hline & $\begin{array}{l}\text { Mediana } \\
\text { IQR }\end{array}$ & $\begin{array}{l}\text { Mediana } \\
\text { IQR }\end{array}$ & Z & $\begin{array}{l}\text { Mediana } \\
\text { IQR }\end{array}$ & $\begin{array}{l}\text { Mediana } \\
\text { IQR }\end{array}$ & $\mathrm{Z}$ \\
\hline $\begin{array}{l}\text { Sentimientos y } \\
\text { actitudes }\end{array}$ & $\begin{array}{l}7.50 \\
5.00\end{array}$ & $\begin{array}{l}4.00 \\
4.00\end{array}$ & $-3.643 * * *$ & $\begin{array}{l}9.00 \\
5.00\end{array}$ & $\begin{array}{l}4.00 \\
4.00\end{array}$ & $-2.585^{*}$ \\
\hline Vida social & $\begin{array}{l}1.00 \\
2.00\end{array}$ & $\begin{array}{l}.00 \\
1.00\end{array}$ & $-2.555^{*}$ & $\begin{array}{l}4.00 \\
5.00\end{array}$ & $\begin{array}{l}2.00 \\
3.00\end{array}$ & ns \\
\hline Economía & $\begin{array}{l}.00 \\
1.00\end{array}$ & $\begin{array}{l}.00 \\
1.00\end{array}$ & $-2.070^{*}$ & $\begin{array}{l}.00 \\
2.00\end{array}$ & $\begin{array}{l}.00 \\
1.00\end{array}$ & ns \\
\hline $\begin{array}{l}\text { Relación matri- } \\
\text { monial }\end{array}$ & $\begin{array}{l}1.50 \\
2.00\end{array}$ & $\begin{array}{l}.00 \\
1.00\end{array}$ & $-2.910 * *$ & $\begin{array}{l}2.00 \\
4.00\end{array}$ & $\begin{array}{l}.00 \\
3.00\end{array}$ & $-2.401 *$ \\
\hline $\begin{array}{l}\text { Relación entre } \\
\text { hermanos }\end{array}$ & $\begin{array}{l}3.50 \\
3.00\end{array}$ & $\begin{array}{l}2.00 \\
3.00\end{array}$ & $-2.965^{* *}$ & $\begin{array}{l}4.00 \\
3.00\end{array}$ & $\begin{array}{l}2.00 \\
3.00\end{array}$ & $-2.041 *$ \\
\hline $\begin{array}{l}\text { Dificultad de } \\
\text { vivir con él }\end{array}$ & $\begin{array}{l}1.00 \\
1.00\end{array}$ & $\begin{array}{l}.00 \\
1.00\end{array}$ & $-2.236^{*}$ & $\begin{array}{l}1.00 \\
0.00\end{array}$ & $\begin{array}{l}1.00 \\
1.00\end{array}$ & ns \\
\hline $\begin{array}{l}\text { Influencia sobre } \\
\text { la familia }\end{array}$ & $\begin{array}{l}.00 \\
.00\end{array}$ & + & $-2.000 *$ & $\begin{array}{l}1.00 \\
1.00\end{array}$ & + & $-2.236^{*}$ \\
\hline
\end{tabular}

$*=\mathrm{p}<.05 ; * *=\mathrm{p}<.01 ; * * *=\mathrm{p}<.001$

Sin embargo, las familias de los niños con TDAH-C con $\mathrm{PC}$ presentan resultados en general menores. Concretamente, aparecen mejoras significativas entre el pretest y el postest en sentimientos y actitudes $(Z=-2.585 ; \mathrm{p}=010)$, en relación matrimonial $(Z=-2.401 ; p=016)$, en relación entre hermanos $(Z=$ $-2.041 ; \mathrm{p}=041)$, $\mathrm{y}$ en influencia negativa sobre la familia $(\mathrm{Z}=$ $-2.236 ; \mathrm{p}=025)$.

Por último, los resultados de las comparaciones entre las familias de niños TDAH-C con y sin PC asociados en las variables que componen el cuestionario de impacto en los dos momentos analizados arrojan diferencias significativas únicamente en la variable vida social en el postest $(U=37.000$; $\mathrm{p}=023)$.

\section{Discusión}

En relación al primer objetivo planteado en este estudio, los resultados son claros y ponen de manifiesto el efecto beneficioso para el conjunto de la familia de la intervención multicomponente implementada con los niños, sus padres y sus profesores. De hecho, el tratamiento afecta positivamente a todos los aspectos familiares analizados. Así, los sentimientos y actitudes de los padres son más positivos hacia el hijo con TDAH. Mejora claramente la vida social e incluso la economía familiar. Se producen cambios positivos igualmente en las rela- ciones dentro de la familia, tanto entre los hermanos como entre el matrimonio. Por último, los padres consideran que, tras el tratamiento es más fácil vivir con su hijo con TDAH y que éste ejerce una influencia mejor sobre la familia. Los avances son tan significativos que llegan incluso a diferenciar en la evaluación de postest a los grupos tratado y no tratado en sentimientos y actitudes de los padres, relación matrimonial e influencia negativa sobre la familia. En el mismo sentido, desaparecen las diferencias del pretest en la relación entre hermanos.

En cuanto al segundo objetivo, relativo a si la presencia de problemas de conducta asociados al TDAH-C modularía los resultados de la intervención sobre estas mismas variables familiares, los resultados son claramente afirmativos. El tratamiento es beneficioso en ambos casos, pero en distinta medida. Además, el grupo TDAH-C sin PC asociados mejora significativamente en todas las variables analizadas tras el tratamiento, mientras que el grupo con PC mejora sólo en sentimientos y actitudes de los padres, relación matrimonial, relación entre hermanos e influencia negativa sobre la familia. El grado diferente de respuesta al tratamiento hace que ambos grupos que se mostraban iguales en el pretest sean diferentes en el postest en vida social.

Entre las aportaciones realizadas por nuestro estudio destaca la coordinación de tres programas paralelos donde se implicaba al niño y a sus dos contextos de desarrollo próximos. Otro de los aspectos destacables es el diseño de un paquete multicomponente que combina las principales técnicas que han mostrado gran eficacia tanto sobre la sintomatología básica del TDAH como sobre las manifestaciones asociadas. El programa incluye procedimientos de modificación de conducta, entrenamiento en estrategias cognitivas de autocontrol, resolución de problemas y control de la ira, habilidades sociales y modificaciones académicas. En este sentido, nuestro trabajo va más allá del uso de intervenciones que normalmente tienden a enfatizar el control conductual del niño con TDAH, omitiendo aspectos tan importantes como la adaptación socioemocional del niño y los que le rodean, aspecto que ha sido tenido en cuenta en esta intervención. Destaca, por tanto, la validez ecológica de la intervención. Su objetivo es ampliar las competencias de los "mediadores" (padres y profesores) y al mismo tiempo conseguir la deseable generalización en los contextos próximos de desarrollo del niño de aquellas estrategias y habilidades tratadas directamente con él.

Entre las limitaciones que afectan a nuestra investigación destacaríamos básicamente tres por su relevancia. La primera de ellas está relacionada con el tamaño muestral. El reducido número de participantes aconseja la utilización de análisis estadísticos no paramétricos que tienen un menor poder de discriminación. Una segunda limitación se refiere a la naturaleza puramente descriptiva de nuestros hallazgos. Para aportar una panorámica más completa y significativa habría que determinar hasta qué punto los factores familiares evaluados actúan 
como variables mediadoras, propiciando el cambio conductual y socio-emocional de los niños tratados. Por último, carecemos de información sobre la posible mejora obtenida en el seguimiento de estos niños. Cabe hipotetizar que el carácter multicomponencial de la intervención, su implementación en un entorno natural y la coordinación estrecha entre todos los participantes, aumenta las probabilidades de que los beneficios sobre la familia a largo plazo se mantengan.

Se puede concluir la eficacia de la intervención realizada sobre el negativo impacto familiar del TDAH-C, aunque no es suficiente. El TDAH, es un trastorno evolutivo, esencialmente crónico, por lo que un factor fundamental es que la familia adopte ante este trastorno una actitud de afrontamiento. Apostamos en este sentido por una colaboración estrecha entre la familia, las instituciones educativas y sanitarias y la sociedad en general. Como otros autores (Chronis et al., 2006; Hinshaw, 2007; Pelham et al., 2005) apostamos por la superación de planteamientos reduccionistas y parciales, para acogernos a un enfoque contextualizado y multicomponente que ponga el énfasis en la prevención, el papel activo de los padres y de los profesores y la intervención multimodal, destacando además la importancia de su mantenimiento a lo largo del tiempo.

Finalmente, aunque este y otros trabajos en la misma línea dan soporte a la implementación de intervenciones psicosociales para niños con TDAH, sería imprescindible que se tuviera en cuenta las particularidades de cada niño. Existe, mucha variabilidad documentada sobre el grado en el que cada niño mejora como consecuencia de los tratamientos. En nuestro estudio, las mejoras experimentadas por los subgrupos con y sin PC son claramente diferentes, lo que apunta a la necesidad de intervenciones específicas. En esta línea, diferentes trabajos (muchos de ellos basados en el MTA) han investigado posibles mediadores que pueden modular la respuesta individual a la intervención, como los conocimientos, atribuciones y expectativas de los padres, la comorbilidad con otros trastornos, la existencia de una psicopatología de los padres, el estatus socioeconómico e incluso la cultura familiar. La elección del programa de tratamiento para cada caso particular debería partir de un análisis previo de los mismos.

\section{Referencias}

American Psychiatric Association (APA, 1994). Diagnostic and statistical manual of mental disorders DSM-IV. Washington DC: Author.

American Psychiatric Association (2000). Diagnostic and statistical manual of mental disorders. (4th edition, rev.). DSM-IV-TR. Washington DC: Author.

Barkley, R.A. (1987). Defiant children: A clinicians manual for assessment and parent training. New York: Guilford.

Barkley, R.A. (2002). Psychosocial treatments for attentiondeficit/hyperactivity disorder in children. Journal of Clinical Psychiatry, 63, 36-43.
Biederman, J. y Faraone, S. V. (2005). Attention-deficit hyperactivity disorder. The Lancet, 366, 237-248.

Brown, T.E. (2003). Trastornos por déficit de atención $y$ comorbilidades en niños, adolescentes y adultos. Barcelona: Masson.

Conover, W.J. (1999). Practical nonparametric statistics (3rd Ed.). New York: Wiles.

Chronis, A. M., Jones, H. A. y Raggi, V. L. (2006). Evidencebased psychosocial treatments for children and adolescents with attention-deficit/hyperactivity disorder. Clinical Psychology Review, 26, 486-502.

Cunningham, C. E. (2007). A family-centered approach to planning and measurement the outcome of interventions for children with attention-deficit/hyperactivity disorder. Journal of Pediatric Psychology, 32, 676-694.

Donenberg G. y Baker B. L. (1993). The impact of young children with externalizing behaviors on their families. Journal of Abnormal Child Psicolygy, 21, 179-98.

Escobar, R., Soutullo, C. A., Hervás, A., Gastaminza, X., Polavieja, P. y Gilaberte, I. (2005). Worse quality of life for children with newly diagnosed attention- deficit/ hyperactivity disorder, compared with asthmatic and healthy children. Pediatrics, 116, 364-369.

Faraone, S. V., Perlis, R. H., Doyle, A. E., Smoller, J. W., Goralnick, J. J., Holmgren, M. A. y Sklar, P. (2005). Molecular genetics of attention-deficit/hyperactivity disorder. Biological Psychiatry, 57, 1313-1323.

Gadow K.D. y Sprafkin J. (1995). Child symptoms inventories. The revised CSI parent teacher checklist. Stony Brook. New York: Chekmate plus LTD.

Hinshaw, S. P. (2007). Moderators and mediators of treatment outcome for youth with ADHD: Understanding for whom and how interventions work. Journal of Pediatric Psychology, 32, 664-675.

Kaminski, P. L., Jones, H. y Harshaw, A. A. (2004). Low parental warmth and conduct problems in ADHD boys. Poster presented at the 112th annual convention of the American Psychological Association, Honolulu, HI.

Lange, G., Sheerin, D., Carr, A., Dooley, B., Barton, V. y Marshall, D. (2005). Family factors associated with attention deficit hyperactivity disorder and emotional disorders in children. Journal of Family Therapy, 27, 76-96.

Mannuzza, S., Klein, R. G. y Moulton, J.L. (2008). Lifetime criminality among boys with attention deficit hyperactivity disorder: a prospective follow-up study into adulthood using official arrest records. Psychiatry Research, 160, 237-46.

McLaughlin D. y Harrison, C. (2006). Parenting practices of mothers of children with ADHD: the role of maternal and child factors. Child and Adolescent Mental Health, 11, 8288 . 
Miranda, A., Grau, D., Marco, R. y Roselló, B. (2007). Estilos de disciplina en familias con hijos con TDAH: influencia en la evolución del trastorno. Revista de Neurología, 44 (Supl 2), 23-26.

Miranda, A., Marco, R. y Grau, D. (2007). Parenting stress in families of children with attention-deficit/hyperactivity disorder: the impact of ADHD subtype and oppositional defiant disorder comorbidity. In T. Scruggs and M. Mastropieri (Eds). Advances on learning and behavioral disabilities (pp. 139-62). NJ: Elsevier.

Miranda, A., Presentación, M. J., Gargallo, B., Soriano, M., Gil, M. D. y Jarque, S. (1999). El niño hiperactivo (TDAH). Intervención en el aula. Un programa de formación para profesores. Castellón: Universitat Jaume I.

Miranda, A., Presentación, M. J., García, R. y Sieghentaler, R. (2009). Intervention with students with ADHD. Analysis of the effects of a multi-component, multi-contextualized program on academic and socio-emotional adjustment. En T Scruggs and M. Mastropieri (Eds), Advances in behavioral and learning disabilities (V1, 21, pp.227-264). Boston: Elsevier JAI Press.

Miranda, A., Rosel, J., Grau, M. D., y Meliá, A. (en prensa). Understanding discipline in families with children who have attention-deficit/hyperactivity disorder: A structural equation model. Journal of Spanish Psyshology.

Patterson, G.R. (1982). A social learning approach (Vol 3): Coercive family process. Eugene OR: Castalia Publishers.
Pelham, W. E., Massetti, G. M., Wilson, T., Kipp, H., Myers, D., Newman, B. B., Billheimer, S. y Waschbusch, D.A. (2005). Implementation of a comprehensive schoolwide behavioural intervention: the ABC Program. Journal of Attention Disorders, 9, 248-260.

Roselló, B., García, R., Tárraga, M., y Mulas, F. (2003). El papel de los padres en el desarrollo y aprendizaje de los niños con trastorno por déficit de atención con hiperactividad. Revista de Neurología, 79-84.

Sattler, J. M. (1982). The assessment of childrens' intelligence (2nd Ed.). Boston: Allyn Bacon, Inc.

Swensen, A. R., Birnbaum, H. G., Secnik, C., Marychenko, M., Greenberg, P., y Claxton, A. (2003). Attention-Deficit hyperactivity disorder: Increased cost for patients and their families. Journal of American Academy of Child and Adolescent Psychiatry, 42, 1415-1423.

Taylor, E., Chadwick, O., Heptinstall, E. y Danckaerts, M. (1996). Hyperactivity and conduct problems at risk factors for adolescent development. Journal of the American Academy of Child and Adolescent Psychiatry, 35, 1213-36.

Wechsler, D. (1993). Escala de inteligencia para niños revisada. Madrid: TEA.

Fecha de recepción: 26 de junio de 2009

Fecha de aceptación: 16 de julio de 2009 